DE

M E D I C I N A

T R O P I C A L

$\mathrm{DE}$

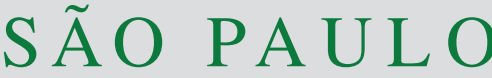

JOURNAL OF THE SÃO PAULO INSTITUTE OF TROPICAL MEDICINE

${ }^{1} \mathrm{FST}$ de Tanger, Equipe de Recherche Biotechnologie et Génie des Biomolécules, Tanger, Morocco

2Institut Pasteur du Maroc, Laboratoire de Biologie Moléculaire, Service de Recherche, Tanger, Morocco

${ }^{3}$ Centre National de l'Energie, des Sciences et Techniques Nucléaires, Unité de Recherches Médicales et Biologiques, Rabat, Morocco

Correspondence to: Imane Chaoui Centre National de l'Energie, des Sciences et Techniques Nucléaires, Unité de Recherches Médicales et Biologiques, BP 1382, RP. 10001, Rabat, Morocco

Tel: +212 537712031

Fax: +212 537711846

E-mail: im_chaoui@yahoo.fr, chaoui@cnesten.org.ma

Received: 7 November 2019

Accepted: 22 April 2020

\section{Frequency of genomic mutations mediating resistance of Mycobacterium tuberculosis isolates to rifampicin in Northern Morocco}

Hind Karimi ${ }^{1,2}$, Amal Oudghiri ${ }^{1,3}$, Latifa En-nanei ${ }^{2}$, Mohammed El Mzibri ${ }^{3}$, Amin Laglaoui ${ }^{1}$, Imane Chaoui ${ }^{-3}{ }^{3}$, Mohammed Abid $^{2}$

\section{ABSTRACT}

Drug resistant tuberculosis (DR-TB) is challenging particularly in developing countries. As such, a previous investigation gave the first insight into the mutational status of the Rifampicin Resistance Determining Region (RRDR) of $r p o B$ gene among a restricted number of MTB patients' residents in the Northern Morocco. The purpose of this study was to investigate rpoB mutation types and frequencies associated with resistance to Rifampicin in a larger panel of MTB patients and to evaluate the usefulness of these mutations to improve the diagnosis of resistance to Rifampicin. A panel of 301 consecutive sputum samples belonging to patients suscpected of having TB from Northern Morocco was collected at the Pasteur Institute of Tangier between 2014-2017. Samples were subjected to conventionel microbiological tests. Evaluation of $r p o B$ muational status was assessed by PCR amplification and sequencing of the RRDR of the $r p o B$ gene. DST results showed that $26.4 \%$ of strains were MDR. Sequencing results reported single point mutations in 36 of $65 \mathrm{RIF}^{\mathrm{R}}$ isolates of which two had two mutations. Aminoacid substitutions in the codon Ser531Leu occurred at the highest frequency (34.46\%). Overall, 10 aminoacid substitutions have been registered, and the H526S substitution was reported for the first time. The present study highlighted that resistance to RIF is a reliable marker of MDR-TB, the common mutations successfully detected in the rpoB 531, rpoB526 and rpoB516 codons provide a foundation for the implementation of molecular approaches such as Hain and GeneXpert as a routine tests to detect DR-TB. However, considerable work is still necessary to identify extensive mutations associated with DR-TB.

KEYWORDS: Mycobacterium tuberculosis. Tuberculosis. MDR-TB. XDR-TB. DNA sequencing. Drug susceptibility testing. Resistance to Rifampicin. rpoB mutations. Morocco.

\section{INTRODUCTION}

Worldwide, tuberculosis (TB) remains a major health problem with nearly 10 million new cases and 1.5 million deaths yearly, of which more than $95 \%$ occur in developing countries where drug surveillance and rapid detection of resistance to anti-TB drugs are uncommon and the lack of proper health care systems often leads to incomplete treatment ${ }^{1}$. The burden of TB has increased with the emergence and dissemination of multidrug-resistant (MDR) and extensively drug-resistant (XDR) Mycobacterium tuberculosis (MTB) strains ${ }^{2}$.

MDR-TB has been defined by the World Health Organization (WHO) as resistance to at least rifampicin (RIF) and isoniazid (INH) ${ }^{1}$, the two most potent drugs used for TB treatment. Moreover, it is widely accepted that most RIF resistant strains are $\mathrm{MDR}^{3}$. As an example, more than $90 \%$ of RIF-resistant isolates are 
also resistant to INH. Therefore, RIF resistance could be considered as a reliable biomarker for MDR-TB detection ${ }^{3}$.

Previous studies demonstrated that in MTB strains, drug resistance is mainly acquired through spontaneous mutations, especially single nucleotide polymorphisms (SNPs) in specific genes. Upon exposure to anti-TB drugs, the resistant populations are selected leading to a sequential accumulation of mutations in the respective genes and consequently generating MDR-TB cases $^{4}$. In this context, RIF resistance is mainly caused by mutations in an 81-bp region of the $r p o B$ gene, named the Rifampicin Resistance Determining Region (RRDR) in up to $96 \%$ RIF-resistant MTB isolates ${ }^{5}$. In this region, mutations in codons 516 , 526 and 531 prevail in most specimens from different populations of different regions and ethnicities ${ }^{3,5-8}$.

Early detection and genotyping of MTB is of great interest to be able to prescribe effective anti-TB therapy, to avoid the transmission and to limit the development of resistance to one or more drugs ${ }^{9}$.

Due to several limitations of traditional techniques, novel approaches and molecular tools for rapid and reliable detection and identification of MTB have been developed and are currently available for TB diagnosis and identification of drug resistant TB (DR-TB) ${ }^{10,11}$. These methods include DNA amplification followed by DNA sequencing, probe hybridization methods, Restriction Fragment Length Polymorphism (RFLP), Single Strand Conformation Polymorphism (SSCP), Heteroduplex analysis and DNA microarrays ${ }^{12,13}$.

Of note, PCR amplification followed by DNA sequencing is mainly the gold technique and the most widely used technique for the identification of mutations in MTB specimens ${ }^{8}$. This technique is the most direct and reliable approach for studying genetic mutations allowing the identification of both, already known and new mutations that are likely to be associated with drug resistance ${ }^{13}$. Automated sequencing has been used by several groups in different clinical settings, allowing the detection of the most frequent mutations, conferring an excellent benefit for patients care $^{7,8,13}$. In Morocco, TB is a major public health concern. The global incidence of all clinical forms of tuberculosis is very high, with nearly 99 new cases per 100,000 inhabitants yearly, corresponding to more than 32,000 new cases per year ${ }^{14}$. According to the last survey on the prevalence of MDR-TB strains, resistance was found in $1 \%$ of new cases and in $11 \%$ of patients with a previous TB history ${ }^{1,15}$

In Morocco, like in other resource-limited countries, WHO guidelines recommend the diagnosis of TB by smear microscopy for all new TB cases, and culture associated with drug susceptibility testing for retreatment cases $^{1,16}$ Drug susceptibility testing is mainly done by the time consuming culture-based proportion method. During the last decade, substantial efforts have been made to meet the requirement of the Moroccan health-care setting for the rapid determination of DR-TB and thereby facilitating the early initiation of appropriate anti-TB treatment ${ }^{14}$. Accordingly, many studies on molecular genotyping of MTB have been conducted and have allowed the introduction of molecular tools in the global management of DR-TB in Morocco, namely GenoType MTBDR ${ }^{\circledR}$ (Hain Lifescience GmbH, Nehren, Germany) and GeneXpert (Cepheid, Sunnyvale, CA, USA) ${ }^{17}$.

In Tangier, the largest city in Northern Morocco, a preliminary study has been conducted to evaluate the contribution of GenoType ${ }^{\circledR}$ MTBDRplus assay in TB and DR-TB diagnosis and has shown that $75 \%$ of RIF $^{R}$ and $100 \%$ of MDR strains harbored mutations in the rpoB gene at codons 516,526 and $531^{17}$. Thus, the present study was planned to investigate the rpoB mutational status associated with resistance to RIF in a large sample to evaluate the usefulness of these mutations as molecular biomarkers for the rapid detection of DR-MTB strains to improve the management of MDR-TB isolates and TB treatment in Northern Morocco.

\section{MATERIALS AND METHODS}

\section{Study setting}

The study was conducted at the Pasteur Institute of Tangier that receives patients from the Tangier region and from all Northern Morocco, mainly Larache and Tetouan cities. Tangier itself is a hot spot area ranking third with TB incidence exceeding 130 cases $/ 100,000$ inhabitants $^{17}$. It is also characterized by an important influx of sub-Saharan immigrants, who are settled there or waiting for immigration to Europe.

Informed consent was obtained from all human adult participants and from the parents or legal guardians of minors together with the approved consent of the Pasteur Institute Ethical Commission (IPM2013-P3).

\section{Sampling and TB diagnosis}

A total of 301 sputum samples were recruited from January 2014 to February 2017. Samples were collected at Tangier's diagnostic centers, Larache and Tetouan cities from patients suspected of TB and sent to the laboratory of Mycobacteriology of the Pasteur Institute. The consecutive sputum samples were decontaminated by the Petroff method and inoculated in Lowenstein-Jensen $(\mathrm{L} / \mathrm{J})$ medium ${ }^{18}$. Direct microscopic examination was performed by the Ziehl-Neelsen 
method $^{19}$. An aliquot of each decontaminated sample was used to extract MTB-DNA for the molecular analysis.

\section{Drug susceptibility testing}

All sputum specimens tested were cultured in L/J medium. The tubes were examined for bacterial growth after $28 \mathrm{~d}$ of incubation at $37^{\circ} \mathrm{C}$. If no growth or little growth was observed, the tubes were left for an extra $14 \mathrm{~d}$.

Drug susceptibility testing (DST) was performed by the proportional method with $\mathrm{L} / \mathrm{J}$ medium ${ }^{20}$. The critical drug concentrations were $0,2 \mu \mathrm{g} / \mathrm{mL}$ for INH and $40 \mu \mathrm{g} / \mathrm{mL}$ for RIF. The critical proportion of resistant bacilli necessary to define a resistant strain is $1 \%$ for the two tested drugs ${ }^{20,21}$.

\section{DNA extraction by the boiling method}

Crude DNA was obtained by scraping a few colonies of $\mathrm{L} / \mathrm{J}$ slants using a sterile disposable loop, then the colonies were added to a screw cap microfuge tube containing $400 \mu \mathrm{L}$ of distilled water. Each sample was first vortexed and then treated by heat shock treatment at $95{ }^{\circ} \mathrm{C}$ for 10 min to inactivate bacteria and release $\mathrm{DNA}^{22,23}$. The DNA thermolysate was used immediately in PCR assays or stored at $-20^{\circ} \mathrm{C}$ until further use.

\section{PCR amplification}

In this study, $r p o B$ gene was amplified by PCR using the TR8 (5'-TGCACGTCGCGGACCTCCA-3') and the TR9 (5'-TCGCCGCGATCAAGGAGT-3') primers. Amplifications were performed in a total volume of $50 \mu \mathrm{L}$ containing $2 \mu \mathrm{L}$ of template DNA, $0.5 \mathrm{pM} / \mu \mathrm{L}$ of each primer, $2.5 \mathrm{mM}$ of each dNTPs (dATP, dCTP, dGTP and dTTP), $25 \mathrm{mM} \mathrm{MgCl}, 1$ unit of Hotstar Taq DNA polymerase (Qiagen, Hilden, Germany) in 1X Taq DNA buffer. Reaction mixtures were first denatured at $94{ }^{\circ} \mathrm{C}$ for $5 \mathrm{~min}$. Then, thirty-five cycles of amplification were performed with denaturation at $94{ }^{\circ} \mathrm{C}$ for $1 \mathrm{~min}$, primer annealing at $58{ }^{\circ} \mathrm{C}$ for $1 \mathrm{~min}$ and DNA extension for 1 min at $72{ }^{\circ} \mathrm{C}$. At the end of the last cycle, the mixtures were incubated at $72{ }^{\circ} \mathrm{C}$ for $7 \mathrm{~min}$. Positive amplifications were confirmed by electrophoresis in $2 \%$ agarose gel with visualization of the $157 \mathrm{bp}$ band.

\section{Sequencing analysis}

PCR products were purified by the ExoSaP-IT clean up system (Thermo Fisher Scientific, Waltham, Massachusetts, USA). Sequencing reaction was performed according to the manufacturer's protocol (BigDye Terminator v1.1
Cycle Sequencing Kit, Applied Biosystems, Thermo Fisher Scientific, Waltham, Massachusetts, USA). Direct sequencing of amplified PCR products was performed with both, the forward (TR8) and the reverse (TR9) primers on an ABI PRISM sequencing apparatus (ABI Prism 3130XL Genetic Analyser, Applied Biosystems, Thermo Fisher Scientific, Waltham, Massachusetts, USA) in the molecular and the functional genomics platforms (UATRS-CNRST, Rabat, Morocco). Resulting chromatograms were manually edited to ensure the sequences accuracy and added to the alignment component of the Molecular Evolutionary Genetics Analysis5 (MEGA5). The mutations found were compared with those included in the TB Drug Resistance Mutation Database ${ }^{24}$.

\section{RESULTS}

A total of 301 clinically symptomatic TB patients were enrolled in this study, but only 208/301 (69.1\%) had positive cultures and thus were subjected to the molecular analysis. Males represent $79 \%$ of the cases vs. $21 \%$ of females, with a sex ratio of 3.7:1.0. The median children-to-adult ratio in this study was 0.04:1.0 (6/149). According to the demographic data, the mean age of patients was 38.68 years with extreme ages at 12 and 72 years old. The distribution of TB cases according to the age showed that TB infection prevails in the age group of $26-45$ years for both, male and female TB patients.

Among the 208 patients, $28.37 \%$ (59/208) were new cases, $14.90 \%$ (31/208) relapsed, $7.21 \%$ (15/208) failed to treatment and $2.88 \%$ (6/208) were under treatment after a period of abandon. Information regarding 97 cases (46.63\%) was missing.

Conventional DST was performed on all the 208 strains for both, RIF and INH. Among them, 67.3\% of the strains were pan-susceptible (140/208) and 26.4\% were MDR (55/208). Mono-resistance to RIF or INH was reported in $4.8 \%(10 / 208)$ and $1.4 \%$ (3/208) of cases, respectively.

PCR assays of the rpoB gene were successful for all RIF-resistant strains (65 cases) which were subjected to DNA sequencing and results are reported in Table 1. Single point mutations resulting in aminoacid change were found in 34 isolates, whereas two isolates had two mutations. Nucleotide substitutions in codon 531were the most recorded (34.46\%). The other hot spot codons, namely 526, 516 and 513 were mutated in $9.23 \%, 6.15 \%$ and $1.54 \%$ of the cases, respectively.

Overall, nine substitutions were recorded in RRDR of the rpoB gene in $55.4 \%$ of TB patients (36/65). Of note, two mutations were found in codon 516: D516Y $(\mathrm{GAC} \rightarrow \mathrm{TAC})$ and $\mathrm{D} 516 \mathrm{~V}(\mathrm{GAC} \rightarrow \mathrm{GTC})$, in one and three 
Table 1 - Frequency of mutations identified by sequencing in the rpoB gene of multidrug-resistant Mycobacterium tuberculosis isolates.

\begin{tabular}{|c|c|c|c|c|c|}
\hline Gene & Position & Type of mutation & Aminoacid changes & $\mathrm{N}^{\circ}$ of isolates & Frequency (\%) \\
\hline \multirow{5}{*}{ rров } & 513 & Substitution of $\mathrm{CAA} \rightarrow \mathrm{GAA}$ & Glutamine $\rightarrow$ Glutamic acid & 1 & 1.54 \\
\hline & 516 & $\begin{array}{l}\text { Substitution of GAC } \rightarrow \text { GTC } \\
\text { Substitution of GAC } \rightarrow \text { TAC }\end{array}$ & $\begin{array}{c}\text { Aspartic } \rightarrow \text { Valine } \\
\text { Aspartic } \rightarrow \text { Tyrosine }\end{array}$ & $\begin{array}{l}3 \\
1\end{array}$ & 6.15 \\
\hline & 526 & $\begin{array}{l}\text { Substitution of CAC } \rightarrow \text { GAT } \\
\text { Substitution of CAC } \rightarrow \text { CTC } \\
\text { Substitution of CAC } \rightarrow \text { TAC } \\
\text { Substitution of CAC } \rightarrow \text { TCC }\end{array}$ & $\begin{array}{c}\text { Histidine } \rightarrow \text { Aspartic acid } \\
\text { Histidine } \rightarrow \text { Leucine } \\
\text { Histidine } \rightarrow \text { Tyrosine } \\
\text { Histidine } \rightarrow \text { Serine }\end{array}$ & $\begin{array}{l}1 \\
3 \\
1 \\
1 \\
\end{array}$ & 9.23 \\
\hline & 531 & $\begin{array}{l}\text { Substitution of TCG } \rightarrow \text { TTG } \\
\text { Substitution of TCG } \rightarrow \text { TGG }\end{array}$ & $\begin{array}{l}\text { Serine } \rightarrow \text { Leucine } \\
\text { Serine } \rightarrow \text { Tryptophan }\end{array}$ & $\begin{array}{c}22 \\
3\end{array}$ & 38.46 \\
\hline & № Mutation & & & 36 & 55.4 \\
\hline
\end{tabular}

cases respectively. In codon 531, two mutations were also registered: S531W (TCG $\rightarrow$ TGG) and S531L (TCG $\rightarrow$ TTG), reported in three and twenty two cases, respectively. As for codon526, four distinct point mutations were reported: $\mathrm{H} 526 \mathrm{D}(\mathrm{CAC} \rightarrow \mathrm{GAT}), \mathrm{H} 526 \mathrm{Y}(\mathrm{CAC} \rightarrow \mathrm{TAC})$, H526L (CAC $\rightarrow$ CTC), and a new mutation H526S $(\mathrm{CAC} \rightarrow \mathrm{TCC}$ ), found in one, one, three and one case, respectively. Only one isolate harbored a mutation in codon 513: Q513E (CAA $\rightarrow$ GAA). Of considerable note, 29 phenotypically RIF $^{\mathrm{R}}$ strains did not harbor any mutation in the RRDR of the $r p o B$ gene $(44.6 \%)$.

\section{DISCUSSION}

This study was conducted with samples from different cities of Northern Morocco, mainly Tangier, considered one of the relevant hot spots of TB in the country. The demographic data revealed that $\mathrm{TB}$ affects men more than women and prevails in adults aged between 26 and 45 years. These data are in agreement with previously reported studies in Morocco and worldwide ${ }^{1,25,26}$, suggesting that men are likely the main vehicle of TB, therefore, special management of male patients will be of great value to prevent dissemination of TB to close contacts, especially women and children. In this context, the situation would be worse with drug resistance to TB. In fact, a number of studies reported age and gender as significant risk factors for spreading of drug resistant $\mathrm{TB}^{27}$.

In Morocco, as in many countries around the world, great efforts have been made for TB diagnosis and treatment, and the main challenge is the steady emergence of drug resistance and dissemination of drug resistance to TB in the population. According to the TB control program recommendations, phenotypic DST should be performed for retreated cases and patients coinfected with HIV for whom the likelihood of acquiring resistance is higher, generating MDR and XDR TB ${ }^{28}$.
It is well known that $\mathrm{TB}$ resistance results from incomplete treatment, often because of an irregular drug supply, inappropriate treatment regimens or poor compliance. Multidrug resistance appears to result from the stepwise acquisition of mutations in specific genes that confer resistance to certain drug targets ${ }^{29}$. For these reasons, considerable efforts have been directed towards ways to speed up the process of susceptibility testing, with limited costs and minimal complexity. To this end, many molecular techniques have been developed and are employed mostly in developed countries with a focus on the screening of drug resistant-associated mutations ${ }^{13,30}$.

In Morocco, many studies have already investigated the prevalence of first line drug resistance associated with genetic mutations in TB patients, mainly from Rabat and Casablanca ${ }^{7,8,13,31}$. Interestingly, Northern Morocco is a very particular region, as it is a gateway to Africa, it is marked by a migration's dynamic fueled by both, the native population migrant workers and foreigner migration especially of sub-Saharan people, generating an upward trend in TB incidence. To the best of our knowledge, only one study was conducted with samples from Northern Morocco and was performed on small sample size to evaluate the contribution of GenoType ${ }^{\circledR}$ MTBDRplus assay in DR-TB diagnosis ${ }^{17}$.

The present study was conducted on $208 \mathrm{~TB}$ samples to identify the main RIF resistance-related mutations and their prevalence. Our findings revealed that $26.4 \%$ of strains were MDR and were mainly isolated from previously treated patients. This prevalence exceeds by far the one previously reported by the TB national program $(11 \%)$ which is very alarming ${ }^{15}$.

In this study, the resistance genotyping was made by PCR-sequencing; this gold method was able to identify widely known drug resistance-associated mutations, as well as novel mutations that could be associated with drug resistance. Of particular interest, $55.38 \%$ of phenotypically RIF $^{R}$ strains harbored mutations in the RRDR, of which 
94.44\% had one single point mutation, whereas the remaining 5.56\% had two SNPs. These results differ from those reported in previous studies conducted in Morocco. Indeed, the presence of mutations in the RRDR region of the $r p o B$ gene was reported in more than $80 \%$ of phenotypically RIF $^{R}$ strains ${ }^{7,11,31}$. This regional variation in the prevalence of point mutations in the RRDR has already been reported and discussed ${ }^{13,32}$.

In this study, most mutations found had been already reported and are in concordance with previous published reports, the most recorded mutation located in the RRDR of the $r p o B$ gene being the substitution Ser531Leu ${ }^{6,7,8,31-33}$.

Overall, mutations in codons 526 and 531 prevail and have been widely reported to correlate with high-level of resistance and have shown cross-resistance between rifabutin and $\mathrm{RIF}^{34,35}$. Mutations in these two sites may occur independently without bias, because mutations in both codons, 531 (Ser $\rightarrow$ Leu) and 526 (His $\rightarrow$ Tyr, His $\rightarrow$ Asp, His $\rightarrow$ Ser and His $\rightarrow$ Leu) have a minimal fitness cost in comparison with the wild-type susceptible strains ${ }^{5,36}$.

Of note, mutations in codons 513 and 516, also named disputed mutations, result in a low-level of resistance to RIF and strains harboring these mutations exhibit high sensitivity to rifabutin ${ }^{6,37}$. Of particular interest, one sample harbored a rare Gln513Glu substitution reported once in a sample from Taiwan ${ }^{38}$.

Despite the large number of mutations already reported in other studies, the evidence of a new mutation in this study, as well as in previous investigations, indicates that mutations continue to arise, probably due to the ability of MTB to adapt to drug exposure ${ }^{39}$. However, it remains to be verified if this mutation is a functional SNP truly associated with RIF resistance in MTB isolates or it is a polymorphism than could be useful for phylogenetic investigations.

Among the phenotypically RIF $^{\mathrm{R}}$ isolates, $44.62 \%$ did not carry any mutations in RRDR of the $r p o B$ gene, this percentage exceeds by far the data reported worldwide (from 1 to $24 \%$ vs $44.62 \%$ in this study). This might be due to (1) the existence of additional mutations outside the 81-bp core region of the $r p o B$ gene, (2) mutations in alternative subunits of RNA polymerase generating resistant phenotypes $^{5,11}$; (3) mutations in other genes (rpoA and rpoC); (4) RIF permeability and (5) some still undefined or efflux pumps mechanisms exhibited by these MTB isolates leading to RIF-resistance mutations in other genes, which ensure the RIF metabolism ${ }^{5,7,8}$.

Overall, our results corroborate earlier data from Morocco regarding the type and frequency of mutations in the $r р о B$ gene supporting that rifampicin-resistant genotypes harbor, in most cases, mutations in critical codons, namely S531L, H526L, D516V.
It is noteworthy that the majority of $r p o B$ gene mutations analyzed by direct sequencing in this study correlated well with RIF resistance-associated mutations targeted by GeneXpert MTB/RIF and Genotype MTBDRplus tests, which supports the suitability and accuracy of these tools to detect RIF-resistant TB in Morocco. Nevertheless, at this stage, the traditional culture and susceptibility testing for RIF resistance are not yet to be replaced. Thus, there are still many challenges and issues to be solved to provide efficient and affordable point-of-care diagnostic tests. Nevertheless, the relatively small number of RIF resistant samples has limited our ability to draw reliable conclusions regarding the frequency and most common types of mutations in the RRDR within MTB isolates from Northern Morocco.

\section{CONCLUSION}

Molecular assays are accurate and rapid screening tools for early detection of DR-TB. The present study focused on the detection of novel and already reported mutations in the RRDR of the $r p o B$ gene within MTB strains prevalent in Northern Morocco. The rapid diagnosis and identification of ubiquitous mutations associated with RIF resistance in MTB are of great value for the effective treatment and monitoring of MDR-TB transmission in Northern Morocco. Future studies should focus on sequencing the entire rро $B$ gene as many studies have documented the occurrence of common and novel mutations outside the RRDR. In addition, novel molecular tests that could identify extensive mutations associated with DR-TB beyond the RRDR are yet to be developed.

\section{ACKNOWLEDGMENTS}

The authors would like to thank the personnel of the Microbiology Department of the Pasteur Institute of Tangier; the personnel of the Lalla Meryem Hospital, Larache; Ben Karrich Hospital, Tetouan and the Diagnostic Center of Tuberculosis, Tangier, for their cooperation.

\section{CONFLICT OF INTERESTS}

None declared.

\section{ETHICAL APPROVAL}

The study protocol was approved by the Ethics Committee of the Pasteur Institute of Morocco, and written informed consent was obtained from each study participant. 


\section{FUNDING}

This research did not receive any specific grant from funding agencies from the public, commercial, or not-forprofit sectors.

\section{REFERENCES}

1. World Health Organization. Guidelines on tuberculosis infection prevention and control, 2019 update. Geneva: WHO; 2019. [cited 2020 Apr 23]. Available from: https://www.who.int/ tb/publications/2019/guidelines-tuberculosis-infectionprevention-2019/en/

2. Streicher EM, Müller B, Chihota V, Mlambo C, Tait M, Pillay M, et al. Emergence and treatment of multidrug resistant (MDR) and extensively drug-resistant (XDR) tuberculosis in South Africa. Infect Genet Evol. 2012;12:686-94.

3. Drobniewski FA, Wilson SM. The rapid diagnosis of isoniazid and rifampicin resistance in Mycobacterium tuberculosis: a molecular story. J Med Microbiol. 1998;47:189-96.

4. Van Rie A, Warren R, Mshanga I, Jordaan AM, van Der Spuy GD, Richardson M, al. Analysis for a limited number of gene codons can predict drug resistance of Mycobacterium tuberculosis in a high-incidence community. J Clin Microbiol 2001;39:636-41

5. Ramaswamy S, Musser J. Molecular genetic basis of antimicrobial agent resistance in Mycobacterium tuberculosis: 1998 update. Tuberc Lung Dis. 1998;79:3-29.

6. Abdelaal A, El-Ghaffar HA, Zaghloul MH, El Mashad N, Badran E, Fathy A. Genotypic detection of rifampicin and isoniazid resistant Mycobacterium tuberculosis strains by DNA sequencing: a randomized trial. Ann Clin Microbiol Antimicrob. 2009;8:4.

7. Kourout M, Chaoui I, Sabouni R, Lahlou O, El Mzibri M, Jordaan AM, et al. Molecular characterisation of rifampicin-resistant Mycobacterium tuberculosis strains from Morocco. Int J Tuberc Lung Dis 2009;13:1440-2.

8. Zakham F, Chaoui I, Echchaoui AH, Chetioui F, Elmessaoudi MD, Ennaji MM, et al. Direct sequencing for rapid detection of multidrug resistant Mycobacterium tuberculosis strains in Morocco. Infect Drug Resist. 2013;6:207-13.

9. Chang KC, Yew WW. Management of difficult multidrug-resistant tuberculosis and extensively drug-resistant tuberculosis: update 2012. Respirology. 2013;18:8-21

10. Reller LB, Weinstein MP, Woods GL. Susceptibility testing for mycobacteria. Clin Infect Dis. 2000;31:1209-15.

11. Sabouni R, Kourout M, Chaoui I, Jordaan A, Akrim M, Victor TC, et al. Molecular analysis of multidrug resistant Mycobacterium tuberculosis isolates from Morocco. Ann Microbiol. 2008;58:749-54.

12. Neonakis IK, Gitti Z, Krambovitis E, Spandidos DA. Molecular diagnostic tools in mycobacteriology. J Microbiol Methods. 2008;75:1-11

13. Chaoui I, Abid M, El Messaoudi MD, El Mzibri M. Detection of Mycobacterium tuberculosis and drug resistance: opportunies and challenges in Morocco. In: Cardona PJ, editor. Understanding tuberculosis: global experiences and innovative approaches to the diagnosis. Rijeka: InTech; 2012. p.471-510.

14. Morocco. Ministère de La Santé. Direction de l'Épidémiologie et de Lutte contre les Maladies. Bull Epidemiol Santé Publique. 2018;56(76):1-53.

15. World Health Organization. Maroc: profil de tuberculose. [cited 2020 Apr 23]. Available from: https://extranet.who.int/sree/ Reports?op=Replet\&name=\%2FWHO_HQ_Reports\%2FG2 $\% 2$ FPROD $\% 2$ FEXT $\% 2$ FTBCountryProfile $\& I S O 2=\mathrm{MA} \& \mathrm{~L}$ $\mathrm{AN}=\mathrm{FR} \&$ outtype $=$ pdf

16. World Health Organization. Interim recommendations for the surveillance of drug resistance in tuberculosis: may 2007. Geneva: WHO; 2007. [cited 2020 Apr 23]. Available from: http://whqlibdoc.who.int/hq/2007/WHO_HTM_ TB_2007.385_eng.pdf

17. Karimi H , En-Nanai L, Oudghiri A, Chaoui I, Laglaoui A, Bourkadi JE, et al. Performance of GenoType MTBDRplus assay in the diagnosis of drug-resistant tuberculosis in Tangier. Morocco. J Glob Antimicrob Resist. 2018;12:63-7.

18. Tripathi K, Tripathi PC, Nema S , Shrivastava AK , Dwiwedi K , Dhanvijay AK. Modified Petroff's method: an excellent simplified decontamination technique in comparison with Petroff's method. Int J Recent Trends Sci Technol. 2014;10:461-4.

19. Singhal R, Myneedu VP. Microscopy as a diagnostic tool in pulmonary tuberculosis. Int J Mycobacteriol. 2015;4:1-6.

20. David H, Lévy-Frebault V, Thorel MF. Méthodes de laboratoire pour mycobactériologie clinique. Paris : Institut Pasteur ; 1989.

21. Canetti G, Froman S, Grosset J, Hauduroy P, Langerova M, Mahler HT, et al. Mycobacteria: laboratory methods for testing drug sensitivity and resistance. Bull World Health Org. 1963;29:56578 .

22. Aldous WK, Pounder JI, Cloud JL, Woods GL. Comparison of six methods of extracting M. tuberculosis DNA from processed sputum for testing by quantitative real-time PCR. J Clin Microbiol. 2005;43:2471-3.

23. Chaoui I, Sabouni R, Kourout M, Jordaan AM, Lahlou O, Elouad $\mathrm{R}$, et al. Analysis of isoniazid, streptomycin and ethambutol resistance in Mycobacterium tuberculosis isolates from Morocco. J Infect Dev Ctries. 2009;3:278-84.

24. Sandgren A, Strong M, Muthukrishnan P, Weiner BK, Church GM, Murray MB. Tuberculosis Drug Resistance Mutation Database. PLoS Med. 2009;6:e1000002.

25. Marçôa R, Ribeiro AI, Zão I, Duarte R. Tuberculosis and gender: factors influencing the risk of tuberculosis among men and women by age group. Pulmonology. 2018;24:199-202. 
26. Wood R, Liang H, Wu H, Middelkoop K, Oni T, Rangaka MX, et al. Changing prevalence of tuberculosis infection with increasing age in high-burden townships in South Africa. Int J Tuberc Lung Dis. 2010;14:406-12

27. Merza MA, Farnia P, Tabarsi P, Khazampour M, Masjedi MR, Velayati AA. Anti-tuberculosis drug resistance and associated risk factors in a tertiary level TB center in Iran: a retrospective analysis. J Infect Dev Ctries. 2011;5:511-9.

28. Morocco. Ministère de la Santé. Direction de l'Épidémiologie et de Lutte contre les Maladies. Guide de la lutte antituberculeuse à l'usage des professionnels de la santé. $3^{\text {rd }}$ ed. Rabat : Ministère de la Santé; 2011.

29. Heym B, Honoré N, Truffot-Pernot C, Banerjee A, Schurra C, Jacobs WR Jr, et al. Implications of multidrug resistance for the future of short-course chemotherapy of tuberculosis: a molecular study. Lancet. 1994;344:293-8.

30. Nguyen TN, Berre VA, Banuls AL, Nguyen TV. Molecular diagnosis of drug-resistant tuberculosis: a literature review. Front Microbiol. 2019;10:794.

31. Oudghiri A, Chaoui I, El Mzibri M. Molecular epidemiology of tuberculosis: a review of tools and applications. J Infect Dis Ther. 2018;6:386.

32. Telenti A, Imboden P, Marchesi F, Lowrie D, Cole S, Colston MJ, et al. Detection of rifampicin-resistance mutations in Mycobacterium tuberculosis. Lancet. 1993;341:647-50.

33. Aziz MA, Wright A, Laszlo A, De Muynck A, Portaels F, Van Deun A, et al. Epidemiology of antituberculosis drug resistance (the Global Project on Anti-tuberculosis Drug Resistance Surveillance): an updated analysis. Lancet. 2006;368:2142-54.

34. Zhang Y, Jacobs W Jr, Vilchèze C. Mechanisms of drug resistance in Mycobacterium tuberculosis. In: Cole S, Eisenach K, McMurray D, Jacobs W Jr, editors. Tuberculosis and the tubercle bacillus. Washington: ASM Press; 2015. p.115-40.

35. Billington OJ, McHugh TD, Gillespie SH. Physiological cost of rifampin resistance induced in vitro in Mycobacterium tuberculosis. Antimicrob Agents Chemother. 1999;43:1866-9.

36. Gagneux S, Long CD, Small PM, Van T, Schoolnik GK, Bohannan BJ. The competitive cost of antibiotic resistance in Mycobacterium tuberculosis. Science. 2006;312:1944-6.

37. Williams DL, Spring L, Collins L, Miller LP, Heifets LB, Gangadharam PR, et al. Contribution of rpoB mutations to development of rifamycin cross-resistance in Mycobacterium tuberculosis. Antimicrob Agents Chemother. 1998;42:1853-7

38. Prammananan T, Cheunoy W, Taechamahapun D, Yorsangsukkamol J, Phunpruch S, Phdarat P, et al. Distribution of rpoB mutations among multidrug-resistant Mycobacterium tuberculosis (MDRTB) strains from Thailand and development of a rapid method for mutation detection. Clin Mirobiol Infect. 2008; $14: 446-53$.

39. Dookie N, Rambaran S, Padayatchi N, Mahomed S, Naidoo K. Evolution of drug resistance in Mycobacterium tuberculosis: a review on the molecular determinants of resistance and implications for personalized care. J Antimicrob Chemother. 2018;73:1138-51 